\title{
Teoría de la mente y empatía como predictores de conductas disociales en la adolescencia
}

\section{Theory of Mind and Empathy as predictors of antisocial behavior during adolescence}

\author{
Olber Eduardo Arango Tobón, Paula Andrea Montoya Zuluaga, Isabel Cristina Puerta Lopera \\ y José Wilmar Sánchez Duque
}

Fundación Universitaria Luis Amigó y al Centro de Investigaciones de la Provincia de los Religiosos Terciarios Capuchinos, Medellín, Colombia

Disponible online 30 de abril de 2014

\begin{abstract}
Se ha propuesto que las características de la teoría de la mente y la empatía son predictores importantes de trastornos de conducta en la niñez y la adolescencia. Los objetivos del presente estudio fueron comparar un grupo de adolescentes, con características de trastorno disocial y un grupo de adolescentes controles, en pruebas que evalúan teoría de la mente y empatía, con el fin de establecer factores predictivos de riesgo o de protección para el desarrollo de conductas disociales en los adolescentes. Los resultados mostraron diferencias estadísticamente significativas entre ambos grupos, en las pruebas de teoría de la mente y de empatía. Se establecieron como factores protectores la dimensión de la empatía denominada toma de perspectiva y las habilidades del adolescente para interpretar los estados mentales y emocionales de acuerdo al Test de la lectura de la mirada.
\end{abstract}

Palabras Clave: Trastorno de Conducta Disocial; Adolescencia; Teoría de la Mente, Empatía.

It has been proposed that the characteristics of theory of mind and empathy are important predictors of behavioural disorders during childhood and adolescence. This study compared a group of teenagers with the characteristics of antisocial behavior disorder and a group of teenagers as controls in terms of their performance on tests assessing the theory of mind and empathy, with the further aim of establishing risk and protective factors predictive of the development of antisocial behavior during adolescence. There were significant statistical differences between the two groups on the theory of mind and empathy tests. The dimension of empathy known as as perspective taking as well as the adolescent's skills in understanding mental and emotional states were established as protective factors according to the Reading the Mind in the Eyes Test.

Key words: Behavior Disorders in Teens; Disocial; Empathy Theory of Mind.

Correspondencia: Olber Eduardo Arango Tobón. Facultad de Psicología y Ciencias Sociales. Fundación Universitaria Luis Amigó. Transversal 51 ${ }^{a}$ \#67B90 Medellín - Colombia. teléfono: + 57(4) 4487666 ext. 9558 +57(4) 314619 7069. E-mail: olber.arangoto@amigo.edu.co - duarango@hotmail.com. E- mail de los otros autores: Isabel Cristina Puerta Lopera: ipuerta@funlam.edu.co, José Wilmar Sánchez Duque: jwsanchez@funlam.edu. co, Paula Andrea Montoya Zuluaga: paula.montoyazu@amigo.edu.co

Este proyecto fue financiado por la Fundación Universitaria Luis Amigó y Centro de Investigaciones de la Provincia de los Religiosos Terciarios Capuchinos, Medellín, Colombia. 
En los últimos años el estudio de la empatía y la teoría de la mente en trastornos de conducta en niños y adolescentes, ha sido un tema de central importancia para investigadores interesados en las descripciones emocionales, cognitivas y comportamentales en estos trastornos (Baron-Cohen, O'Rioldan, Stone y Plaisted, 1999; Ellis, 1982; Dolan y Fullam, 2004; Ekman y Freisen, 1975; Fairchild, Van Goozen, Calder, Stollery y Goodyer, 2009; Miller y Eisenberg, 1988). Estos investigadores consideran que los retrasos o déficits en el desarrollo de la empatía y teoría de la mente pueden estar asociados con cuadros clínicos más graves y que su normal desarrollo puede generar procesos que inhiben conductas agresivas y antisociales durante la niñez y la adolescencia.

Se plantea que en la etiología del trastorno disocial (TD), las formas más severas y persistentes se desarrollan en individuos varones con alteraciones en su neurodesarrollo, mostrando conductas impulsivas, agresivas y un bajo coeficiente intelectual verbal (Baker, 2013; Hill, 2002; Murray y Farrington, 2010). El TD es definido como un patrón de conducta que viola los derechos de los otros o transgrede importantes normas sociales (American Psychiatry Association, 2000). Las conductas disociales se caracterizan también por una pobre adaptación social que puede ser resultado de factores genéticos, alteraciones en el procesamiento cognitivo y emocional y/o interacciones con entornos maladaptativos que generan aprendizajes sociales inadecuados (Mizock y Harkins, 2011; Weiss, Dodge, Bates y Pettit, 1992). Los problemas de conducta durante la adolescencia han sido asociados también a déficits neuropsicológicos relacionados con el funcionamiento de los lóbulos frontales que intervienen en la capacidad para planificar conductas, predecir los efectos de las propias conductas sobre los demás, dichos déficits generan alteraciones para procesar la información emocional y problemas significativos en la cognición social y el desarrollo empático (Ellis, 1982; Dolan y Fullam, 2004; Jones, Forster y Skuse, 2007; Miller y Eisenberg, 1988; Peets, Hodges y Salmivalli, 2010). Algunas investigaciones se han centrado en el estudio de la empatía como factor inhibidor de conductas disociales y agresivas.

Ellis (1982), planteó la importancia que juega la empatía en el desarrollo de la conducta antisocial y agresiva. En su estudio, usó una muestra de 331 jóvenes delincuentes y 64 jóvenes no delincuentes. El grupo de jóvenes delincuentes fue clasificado según el tipo de delincuencia (137 psicopáticos, 95 neuróticos y 100 tipo subcultural) y también el tipo de agresión (81 delincuentes no agresivos, 159 agresores a personas y 91 agresores a la propiedad). Los resultados de este estudio señalaron retrasos o interrupciones en el desarrollo empático en el grupo de jóvenes delincuentes. De los tres grupos de delincuentes, el grupo neurótico fue el menos empático, seguido por el grupo psicopático y el grupo subcultural. Por otro lado, encontró que el grupo de delincuentes con agresiones a personas son menos empáticos que el grupo de delincuentes con agresiones contra la propiedad. La importancia de este estudio radica en la relación que hace el autor entre agresión/conducta antisocial y empatía, que soporta la concepción de la empatía como una respuesta emocional inhibidora de conductas antisociales durante la adolescencia.

En esta misma línea, Miller y Einsenberg (1988), plantearon que la correlación entre empatía y conductas antisociales y agresivas es negativa, es decir, en la medida que existen bajos niveles de empatía, aparecen conductas exteriorizadas en los sujetos. En su estudio de meta-análisis incluyeron investigaciones empíricas asociadas con las relaciones entre psicopatología de la empatía y el abuso físico e investigaciones que relacionaban empatía y conductas agresivas. Concluyeron que dichas relaciones estadísticas están afectadas por los métodos de medición que se empleen para evaluar la empatía (historias/ dibujos, cuestionarios de auto-informe, identificación de estados emocionales en otros y métodos experimentales de inducción empática). Los autores encontraron específicamente que las relaciones negativas más significativas entre empatía y agresión se hallaron en estudios que usaban técnicas de cuestionarios (auto-informes) para la evaluación de la empatía; aunque el resto de métodos de evaluación también mostró relaciones negativas pero no significativas. Este estudio proporciona evidencias que indican el papel de la empatía como inhibidor de conductas agresivas y antisociales, no obstante, plantean la necesidad de tener en cuenta diferentes factores para entender mejor las relaciones entre las respuestas empáticas y conductas exteriorizadas, tales como la edad, la existencia de diferentes tipos de trastornos de la conducta durante la infancia y la adolescencia y las diferencias individuales en cuanto al desarrollo empático de los individuos.

Otros estudios plantean además, que los procesos implicados en la cognición social y el coeficiente intelectual (CI) tienen también una fuerte relación en la expresión de conductas agresivas y disociales durante la infancia y la adolescencia. En esta línea, Jones et al. (2007) propusieron que los adolescentes con TD presentaban bajo coeficiente intelectual y bajas competencias verbales. También, un significativo deterioro para reconocer emociones en los demás y un déficit en la correcta interpretación de la mirada en las otras personas. En su estudio evaluaron los déficits asociados a la cognición social en un grupo de 15 adolescentes con TD y un grupo control de 22 adolescentes sin TD. Los hallazgos de este estudio mostraron diferencias estadísticamente significativas en el funcionamiento intelectual y verbal, obteniendo peores resultados el grupo de adolescentes con TD. De igual manera, se encontró que estos adolescentes tienen dificultades para interpretar adecuadamente las expresiones faciales de la ira y el asco y una marcada dificultad para "leer" la mirada de los otros. Es decir, mostraron déficits para inferir los estados emocionales de otros de acuerdo a la expresión de su mirada. Los resultados de este estudio son interesantes, especialmente por las dificultades encontradas en la interpretación de la mirada, ya que esta habilidad juega un importante papel en las interacciones sociales porque cumple 
con una función comunicativa en las que se transmiten señales sociales y se refuerzan o castigan conductas socialmente aceptables o reprobables (Blair, 2003; Fairchild et al., 2009). Es consistente el grupo de investigaciones que han señalado la importancia del reconocimiento de las emociones en los otros como factor esencial para establecer procesos empáticos y que la existencia de déficits en estas habilidades aumentan la probabilidad de aparición de problemas de conducta durante la niñez y la adolescencia.

Fairchild et al. (2009) estudiaron el reconocimiento de la expresión facial en adolescentes con TD con altos niveles de agresión y conductas antisociales. Se compararon los resultados en el test de reconocimiento facial de Benton (Benton, Hamsher, Varney y Spreen, 1983) y la tarea del hexágono emocional (Ekman y Freisen, 1975) entre un grupo de adolescentes TD de inicio temprano de 42 sujetos, adolescentes con TD de inicio tardío de 39 sujetos y un grupo control de 42 sujetos. Los autores encontraron déficits en el reconocimiento de la ira, el asco, el miedo y la alegría en el grupo de adolescentes con TD, tanto en el grupo de inicio temprano como en el de inicio tardío, en comparación con el grupo control. Estos resultados apoyan los hallazgos del estudio realizado por Jones et al. (2007) y señalan las posibles deficiencias de los adolescentes con TD para comprender las emociones y sentimientos de los demás; afectando a las habilidades sociales para la interacción interpersonal, la teoría de la mente, la sensibilidad emocional y los procesos empáticos.

Otros autores también han encontrado resultados similares. Gómez, Arango, Molina y Barceló (2010), partiendo del supuesto de los déficits en la cognición social y los déficits en la empatía en el TD, estudiaron el procesamiento emocional y empático en esta población. En su estudio, se usó una muestra de 60 menores infractores entre 10 y 16 años de edad (30 TD y 30 no TD), administraron la prueba de la lectura de la mirada (The Child Eyes Test; Baron-Cohen, Wheelwright, Hill, Raste y Plumb, 2001) y el Test de meteduras de pata (Faux Pas Task; Stone, Baron-Cohen y Knight, 1998; Gregory et al., 2002) con el fin de comparar las actuaciones de ambos grupos en estas tareas. Los resultados de esta investigación mostraron diferencias estadísticamente significativas entre los grupos. Concretamente, el grupo de adolescentes infractores con TD mostró una peor actuación en la prueba de meteduras de pata. Esto sugiere que la condición diagnóstica tiene una relación directa en la forma en que los adolescentes con TD interactúan socialmente, atribuyen estados mentales a otras personas y situaciones de manera equivocada y presentan deficiencias en la sensibilidad social para comprender y darle importancia a los sentimientos y emociones de los demás.

En otro grupo de investigaciones, se han encontrado déficits significativos en la teoría de la mente en niños con conductas agresivas y adolescentes con trastorno disocial. La teoría de la mente es una dimensión de la cognición social y se refiere a la capacidad de interpretar la conducta de los otros en un contexto mental, es decir, la habilidad del sujeto para atribuir pensamientos, sentimientos, ideas e intenciones de los demás y emplear dicha habilidad para anticipar e influir en la conducta de los otros (Sharp, 2008). De acuerdo con lo anterior, hay estudios que proponen la hipótesis de la existencia de deficiencias en la comunicación emocional y sensibilidad social en niños y adolescentes con problemas de conducta. Sharp (2008), evaluó las realizaciones de pre-adolescentes y jóvenes adolescentes con características del trastorno disocial en el Test de la mirada (The Child Eyes Test; Baron-Cohen et al., 2001), junto con la evaluación de su coeficiente intelectual y el informe de sus padres sobre problemas de conducta usando el Cuestionario de fortalezas y dificultades para padres de Goodman (Strengths and Difficulties Questionnarie)(SDQ; Goodman, 1997). La muestra, perteneciente a escuelas públicas de Cambridgeshire (UK), estuvo formada por 79 niños (35 niños y 44 niñas) con una media de edad general de 11 años (11 meses). Los resultados de este estudio en el test de la mirada mostraron que los adolescentes varones obtuvieron puntuaciones más bajas que las mujeres. La edad y el coeficiente intelectual bajo fueron predictores para la presencia de problemas de conducta en los adolescentes en ambos sexos.

En esta misma línea, Peets et al. (2010) plantearon que las habilidades en la teoría de la mente tienen gran validez predictiva a la hora de determinar problemas en la conducta de los niños y adolescentes, relacionados con la agresión y las relaciones interpersonales. En su estudio usaron una muestra de 195 niños (109 niños y 86 niñas) entre 11 y 12 años de edad. Para la evaluación de la cognición social se usó una adaptación del Social-Cognitive Assessment Profile (SCAP; Hughes, Meehan y Cavell, 2004), que evalúa la capacidad para el procesamiento de información emocional en niños y adolescentes. Los resultados de este estudio mostraron una tendencia cognitiva y afectiva de niños y niñas en el uso de estrategias cognitivas para resolver situaciones sociales mediante conductas agresivas y hostiles y valorando positivamente dichas estrategias como métodos efectivos para lograr objetivos sin importar las señales emocionales que los otros niños manifestaban. De acuerdo a lo anterior, algunos autores plantean que los déficits en la interpretación y lectura de las expresiones faciales de las emociones, son factores predictivos de conductas disociales o por lo menos características de individuos con bajas competencias y habilidades sociales (Fairchild et al., 2009; Scourfield, Martin, Eley y McGuffin, 2004).

Los procesos involucrados en la teoría de la mente, pueden considerarse como un importante factor predictivo para el desarrollo de conductas antisociales y psicopáticas en la adultez; dichas habilidades son un prerrequisito para generar respuestas empáticas, las cuales facilitan la inhibición de conductas antisociales (Ellis, 1982; Miller y Eisenberg, 1988; Sharp, Croudace y Goodyer, 2007). Estas respuestas empáticas promueven conductas positivas como la ayuda a otros y previenen o reducen 
las conductas antisociales, incluyendo la agresión y la delincuencia (Eisenberg, 2009; Jollife y Farrington, 2004; Jones, Happé, Gilbert, Brunnett y Viding, 2010).

Estos hallazgos han sido estudiados en adultos con trastorno antisocial de la personalidad. Dolan y Fullam (2004) estudiaron la teoría de la mente como un factor implicado en la patogénesis de la conducta antisocial. En su estudio usaron una muestra de 89 hombres que cumplían criterios clínicos para el trastorno de personalidad antisocial (estos 89 sujetos fueron clasificados según criterios de psicopatía, con puntaje de corte 18 en checklist del DSM IV) y 20 controles (pareados por edad (entre 18 y 55 años) y coeficiente intelectual). Se aplicó The Interpersonal Reactivity Index (IRI; Davis, 1980), que evalúa cuatro dimensiones de la empatía (Toma de perspectiva, Fantasía, Preocupación empática y Malestar personal); el Test de falsas creencias; el Test de meteduras de pata (Baron-Cohen, et al., 1999) y el Test de expresión facial emocional (BaronCohen, Wheelwright y Jolliffe, 1997). Los resultados de este estudio no mostraron diferencias significativas entre el grupo control y los grupos con trastorno antisocial, relacionadas con las tareas que medían teoría de la mente. No obstante, se pudo corroborar que dentro del grupo con trastorno antisocial, aquellos que cumplían el criterio de psicopatía (entendida esta como el cumplimiento de los criterios diagnósticos del trastorno de personalidad antisocial según el DSM IV) obtuvieron peores resultados en el test de meteduras de pata. Por otra parte, en el test de expresión emocional el grupo con trastorno antisocial no psicopático, mostró mayores limitaciones en la tarea de reconocer emociones básicas. Finalmente este estudio concluyó, que los déficits en la teoría de la mente en estos sujetos están relacionados más por su incapacidad de asumir la perspectiva del otro que por su falta de preocupación por las conductas dañinas a los demás.

En síntesis, las investigaciones en torno al estudio de la empatía, el CI y la cognición social, en particular la dimensión de la teoría de la mente, muestran un abordaje que ha posibilitado la comprensión de estas variables emocionales, cognitivas y comportamentales en trastornos de conducta durante la infancia y la adolescencia. Asimismo, señalan la importancia que tiene un adecuado desarrollo de la empatía, la teoría de la mente y funcionamiento intelectual como factores predictores e inhibidores de dichos trastornos o, por otro lado, como factores de riesgo que podrían relacionarse con la aparición de conductas agresivas y antisociales.

De acuerdo a la revisión de las publicaciones, se observa un limitado grupo de estudios que investigaron la cognición social, la empatía y el CI de forma simultánea en el TD. La propuesta de este estudio es actual y complementa la descripción de la teoría de la mente con dimensiones de la empatía, posibilitando una mejor comprensión de estos constructos en la psicopatología infantil y del adolescente. Otro elemento reseñable de este estudio es que se integra el uso de medidas de ejecución con el uso de medidas de autoinforme para evaluar la empatía y la teoría de la mente, lo cual ha permitido recolectar datos que se validan desde ambas perspectivas.

Se espera que nuestros resultados aporten una mejor comprensión en la forma en que se evalúan y diagnostican a los adolescentes con TD en el contexto latinoamericano, ya que la mayoría de estudios de esta naturaleza se realizaron en contextos anglosajones, dejando de lado determinantes culturales importantes para explicar estos trastornos. Además, este estudio amplía el abordaje del TD desde la dimensión de la cognición social y la empatía como variables que acompañan o puedan predecir el desarrollo de dichos trastornos en nuestro contexto cultural.

El presente estudio comparó adolescentes con características de TD y un grupo control, en términos de sus resultados en el test de la mirada, el test de meteduras de pata, funcionamiento intelectual y algunas dimensiones de la empatía. El primer objetivo del estudio fue comparar los resultados de ambos grupos en pruebas que medían teoría de la mente, CI y empatía. El segundo objetivo, fue establecer factores predictivos de la conducta disocial en los adolescentes.

\section{Participantes \\ Método}

En este estudio se tomó como universo poblacional el número total de adolescentes varones con edades comprendidas entre los 14 y 17 años, de seis instituciones de atención al menor infractor de la Congregación de Religiosos Terciarios Capuchinos de Colombia: Escuela de trabajo Redentor (ETER); Centro de recepción y observación (CETA); Centro de internamiento preventivo Amigoniano (CIPA); Centro de atención al joven Carlos Lleras Restrepo (POLA); Ciudadela los Zagales y Centro Juvenil Amigoniano y dos instituciones de educación básica y media: Colegio Luis Amigó e Instituto técnico San Rafael. De esta forma la población estuvo formada por 1343 adolescentes. Mediante muestreo probabilístico por conglomerados y estratificado por tipo de institución, se seleccionó al azar una muestra de 239 adolescentes varones dividida en dos grupos: 157 adolescentes con características de TD (grupo TD) y 82 adolescentes sin características TD (grupo NO TD). La edad media fue de 16 años $(D T=1.04)$ y de estrato socioeconómico bajo (56.1\%) y medio (40.6\%). En Colombia se entiende el estrato socioeconómico como una clasificación social (Clase Social) que tiene en cuenta el nivel de pobreza de los propietarios de los inmuebles residenciales, la dotación de los servicios públicos y la ubicación del mismo (urbana o rural). Existen seis estratos socioeconómicos. El estrato 1 es el más bajo y el estrato 6 el más alto. Todos los participantes de este estudio aceptaron participar voluntariamente y firmaron consentimiento informado. En todos los casos tanto los adolescentes como sus padres o tutores autorizaron la participación en el estudio. En la Tabla 1, se describen los datos del muestreo y el total de la muestra según el tipo de institución. 
Tabla 1

Muestreo probabilístico por conglomerados del total de la población de instituciones de la Congregación de los Terciarios Capuchinos (Colombia)

\begin{tabular}{llccc}
\hline Institución & Ciudad & $\begin{array}{c}\text { Total } \\
\text { Población }\end{array}$ & $\begin{array}{c}\text { Peso por } \\
\text { institución }\end{array}$ & $\begin{array}{c}\text { Muestra por } \\
\text { institución }\end{array}$ \\
\hline ETER & Bogotá & 236 & .1333 & 42 \\
CETA & Bogotá & 88 & .0497 & 16 \\
CIPA & Bogotá & 174 & .0983 & 31 \\
POLA & Medellín & 145 & .0819 & 26 \\
Cuidadela los Zagales & Manizales & 126 & .0712 & 22 \\
Centro juvenil Amigoniano & Tunja & 165 & .0932 & 29 \\
Colegio Luis Amigó & Medellín & 114 & .0644 & 20 \\
Instituto Técnico San Rafael & Manizales & 295 & .1667 & 53 \\
\multicolumn{1}{c}{ Total } & & 1343 & .7587 & 239 \\
\hline
\end{tabular}

Nota: Se extrajo la muestra con un nivel de confianza del $95 \%$ y un error de muestreo del $5 \%$

\section{Instrumentos}

Mini entrevista neuropsiquiátrica internacional (MINI Kid). Esta prueba se utilizó para determinar si el adolescente tenía o no características del TD. Además, se usaron los criterios del Manual Diagnóstico y Estadístico de los Trastornos Mentales (DSM IV-TR), que indica que el menor de edad que presente tres o más comportamientos que de manera persistente transgreden los derechos básicos de los demás a través de agresión a personas o animales, destrucción de la propiedad ajena, fraude o robo y violaciones graves de las normas. Si el adolescente presentaba tres o más comportamientos de este tipo durante los últimos doce meses y al menos un criterio durante los últimos seis meses se consideraba que tenía características TD. Para confirmar las características TD se usó el módulo Trastorno Disocial de la Mini International Neuropsychiatric Interview (MINI Kid; Sheehan et al., 1997). La MINI Kid es una entrevista diagnostica estructurada de breve duración que explora los principales trastornos psiquiátricos del eje I del DMS IV y el ICD- 10. Estudios de validez y de confiabilidad han comparado la MINI con The Structure Clinical Interview for DSM, Psychiatric Patients (SCID-P) p ara el DSM-III-R y la (Composite International Diagnostic Interview) (CIDI), entrevista estructurada desarrollada por la Organización Mundial de Salud para entrevistadores no clínicos para el ICD-10. Los resultados de estos estudios demuestran que la MINI Kid tiene una puntuación de validez y confiabilidad aceptablemente alto y puede ser administrada en un tiempo mucho más breve (de 11.6 a 18.7 minutos; 15 minutos de promedio) que los instrumentos mencionados. Puede ser utilizada por clínicos después de una breve sesión de entrenamiento. Los entrevistadores no clínicos deben recibir un entrenamiento más intenso.

Test Breve de Inteligencia de Kaufman (K-BIT; Kaufman y Kaufman, 1997). Para la evaluación del funcionamiento y coeficiente intelectual. Es una medida de inteligencia general en niños, adolescentes y adultos de un amplio rango de edad (desde los 4 a los 90 años). Su aplicación es individual y su duración aproximada es de 30 minutos. Está formado por dos subtests: a) Vocabulario, en el que se miden habilidades verbales, desarrollo del lenguaje, formación de conceptos y caudal de información; y b) Matrices, que evalúa habilidades no verbales, como la capacidad de solucionar problemas de razonamiento a través de estímulos visuales tanto figurativos como abstractos. Las puntuaciones que se obtienen tras su aplicación tienen una media de $100(D T=15)$, tanto para los subtests de Vocabulario y Matrices, como para el CI compuesto.

Índice de reactividad interpersonal (Interpersonal Reactivity Index; Davis, 1980). Es una medida de la empatía mediante autoinforme que consta de 28 ítems distribuidos en cuatro sub-escalas de siete ítems cada una, que miden cuatro dimensiones separadas del concepto global de empatía: Toma de Perspectiva, Fantasía, Preocupación Empática y Malestar Personal. Toma de perspectiva mide la habilidad para comprender y asumir el punto de vista de otra persona. Fantasía evalúa la capacidad imaginativa para identificarse y ponerse en el lugar de un personaje ficticio. Preocupación Empática mide la respuesta afectiva de una persona ante dificultades de otras personas, manifestándose en sentimientos de compasión y cariño hacia otros; y finalmente la subescala Malestar Personal mide la experiencia de malestar que siente una personal, ante el estado emocional de otra y la dirige a intentar aliviar su propio estado en lugar de ayudar al otro. Davis (1980), informó que la consistencia interna para las subescalas es de .68 a .79 y su confiabilidad test-retest oscila entre .61 y .81 durante un intervalo de 60 y 75 días. La validez de constructo de las subescalas ha sido corroborada a través de correlaciones con otras medidas de la empatía (Davis, 1983).

Para la evaluación de la teoría de la mente se han utilizado dos instrumentos:

El Test de metedura de pata (Faux Pas Task; Stone, Baron-Cohen y Knight, 1998; Gregory et al., 2002). Este test mide la habilidad para detectar cuándo alguien dice algo inapropiado pero sin mala intención, porque era o podría haber resultado hiriente para la otra persona. Es decir, este test permite establecer la sensibilidad social en las interacciones con otros. Esta capacidad se desarrolla entre los 9 y 11 años. El test consiste en 20 historias basadas en el procedimiento usado por Stone et al. (1998), dentro de las cuales la mitad contiene un Faux pas social (paso en falsa-metedura de pata) y la otra mitad son historias control que contienen un conflicto menor que no constituye un Faux pas. Se le dice al sujeto: "Le voy a leer unas breves historias y hacerle unas preguntas acerca de ellas. Usted tiene una copia de la historia delante suyo así que puede leer mientras yo se las leo y también, volver a leerlas si es que lo necesita". Las historias se presentan una a una, en orden fijo, cada una en hoja aparte. El texto se coloca en frente al participante y el examinador lo lee en voz alta. Existen preguntas de detección del Faux pas y preguntas de memoria que chequean la comprensión de detalles de la his- 
toria. El sistema de puntuación utilizado fue realizado por Stone et al (1998) y se presentan índices de fiabilidad en testretest de .83 y fiabilidad entre evaluadores de .76 (Stone et al, 1998).

El Test de la mirada (The Reading the Mind in the Eyes; Baron Cohen et al., 2001). El estímulo consiste en 36 fotografías de ojos de diferentes actores. Son presentadas una por vez en un orden fijo. La mirada representa estados mentales complejos, definidos por Baron-Cohen et al. (2001) como aquellos que involucran la atribución de una creencia o intención a una persona. Cada estímulo tiene a su alrededor cuatro palabras que describen emociones, dentro de las cuales el participante tiene que elegir aquella que mejor describa lo que la persona está pensando o sintiendo. Se le pide al participante que: "Para cada par de ojos, elija qué palabra mejor describe lo que la persona en la foto está pensando o sintiendo. Ud. puede sentir que más de una palabra es aplicable, pero por favor elija sólo una palabra, la palabra que Ud. considera más adecuada." También se le provee al participante un glosario con todas las palabras, y se les advierte que puede consultarlo en caso de desconocer el significado de alguna de ellas, o pedirle al evaluador que lo haga en voz alta. Se obtiene un punto por cada respuesta correcta; el puntaje máximo total es 36. Los datos de baremación se tomaron del estudio en el contexto latinoamericano en población normal (Román et al., 2012).

\section{Procedimiento}

Una vez seleccionados los individuos mediante muestreo probabilístico, se procedió a realizar las evaluaciones en las instituciones. El equipo de evaluación estuvo conformado por profesionales clínicos de las instituciones e integrantes del grupo de investigación en Neurociencias Básicas y Aplicadas de la Fundación Universitaria Luis Amigó (Funlam), todos ellos fueron entrenados en la aplicación de las pruebas. El protocolo de evaluación fue aprobado por el comité de bioética de la Vicerrectoría de Investigaciones de la Funlam. Una vez obtenida la autorización del joven y sus padres o tutores para participar en el estudio, se comenzó el trabajo de campo en cada una de las instituciones, comenzando con la aplicación de los criterios del DSM IV-TR para el trastorno disocial de la conducta, luego se aplicó la MINI Kid para establecer los grupos según el cumplimiento de los criterios clínicos (TD - NO TD). Finalmente se aplicó el K-BIT, la prueba de empatía y las pruebas de cognición social. A todos los jóvenes participantes tanto del grupo TD como NO-TD, se les explicaron las condiciones de confidencialidad de la información haciendo énfasis en que solamente sería usada con fines científicos y sin repercusiones para sus hojas de vida, nadie en las instituciones usaría sus respuestas individuales con fines diferentes a los científicos y académicos (toda esta información se explicó en el momento de firmar el consentimiento informado, tanto a padres como a los jóvenes). Las evaluaciones tuvieron una duración aproximada de una hora y media y se realizaron en una sola sesión en lugares tranquilos, cómodos y silenciosos.

\section{Análisis estadísticos}

Todos los análisis estadísticos se realizaron mediante el programa informático SPSS para Windows versión 20.0 en español. En primer lugar se realizaron las pruebas de hipótesis de distribución normal de los datos mediante la prueba Kolmogorov-Sminov de acuerdo a los dos grupos (TD y NO TD) de las variables cuantitativas (CI Verbal, CI Matrices, CI Total, meteduras de pata (Faux pas), test de la mirada y las cuatro dimensiones del IRI). Se obtuvieron los descriptivos básicos para cada una de las variables demográficas y las variables relacionadas con el coeficiente intelectual, las dimensiones de la empatía y la teoría de la mente. Para comparar las medias de las pruebas aplicadas en ambos grupos (TD y NO TD), se realizaron pruebas paramétricas ( $t$ de Student) y no paramétricas ( $U$ de Mann Whitney) para establecer las diferencias entre los grupos. Las pruebas paramétricas se aplicaron a los datos cuyas distribuciones eran normales, es decir si el valor $p>.05$, concretamente, las puntuaciones en CI Total y CI Verbal. Como el valor de $p$ para el resto de los datos fue $<.05$, se aplicaron pruebas no paramétricas para la comparación de las medias en estos puntajes en ambos grupos. Finalmente se realizó un análisis de regresión logística con el fin de determinar las relaciones entre la variable dependiente (TD - NO TD) y las variables independientes representadas por las ejecuciones en las pruebas de teoría de la mente, funcionamiento intelectual y las dimensiones del IRI, y poder establecer si eran o no predictivas para el desarrollo de conductas disociales.

\section{Resultados}

La distribución de la muestra en cuanto al estrato socioeconómico señala un mayor porcentaje de sujetos en el grupo TD pertenecientes al estrato bajo (bajo $=120(76.4 \%)$; medio $=34$ $(21.7 \%)$ y alto= $3(1.9 \%)$; mientras que en el grupo NO TD hubo un mayor porcentaje de sujetos en el estrato medio (bajo= $14(17.1 \%) ;$ medio $=63(76.8 \%)$ y alto $=5(6.1 \%)$.

La Tabla 2 contiene las características demográficas de la muestra. Se observan diferencias significativas con relación a la edad $(U=1701.50 ; p<.001)$, la escolaridad $(U=25.42 ; p$ $<.001)$, el CI Total $(t=3.27 ; p<.001)$ y CI Verbal $(t=3.51 ; p$ $<.001)$. Por otro lado, no hubo diferencias en los puntajes del CI Matrices en los grupos. El grupo NO TD obtuvo mejores puntajes de coeficiente intelectual que el grupo TD. No obstante, el grupo TD mostró puntuaciones normales -esperadas para su edad- en el funcionamiento intelectual, teniendo en cuenta que se tomó como referencia para la medición mediante el K-BIT una media de 100 y una desviación estándar de 15 puntos. 
Tabla 2

Características Demográficas

\begin{tabular}{lccccc}
\hline & $\begin{array}{c}\text { TD } \\
(n=157)\end{array}$ & $\begin{array}{c}\text { NO TD } \\
(n=82)\end{array}$ & $\begin{array}{c}t \text { de } \\
\text { Student }\end{array}$ & $\begin{array}{c}U \text { de } \\
\text { Mann } \\
\text { Whitney }\end{array}$ & $p$ \\
\hline Edad & $16.54(0.66)$ & $15.07(0.97)$ & & 1701.50 & $<.001$ \\
Escol & $7.41(2.05)$ & $9.56(1.30)$ & & 25.42 & $<.001$ \\
CI Total & $88.61(18.70)$ & $95.67(14.05)$ & 3.27 & & $<.001$ \\
CI Verbal & $92.04(17.54)$ & $99.90(13.96)$ & 3.51 & & $<.001$ \\
CI Matrices & $92.67(19.20)$ & $96.83(14.94)$ & & 5519.50 & $<.070$ \\
\hline
\end{tabular}

Nota: Todos los datos muestran la media seguido de la desviación estándar entre paréntesis (DE) o la frecuencia y el porcentaje entre paréntesis. ESE = Estrato socioeconómico; Escol = Escolaridad; CI Total $=$ Coeficiente Intelectual Total .

En la Tabla 3, se muestran las medidas obtenidas por ambos grupos en las pruebas que evaluaron cognición social y desarrollo empático.

Como se puede observar (véase Tabla 4), a excepción de la dimensión preocupación empática $(t=1.34 ; p=.018)$ las dife- rencias fueron estadísticamente significativas en el resto de las pruebas. El grupo TD obtuvo puntuaciones significativamente menores en el test de la mirada $(t=7.29 ; p<.001)$, metedura de patas $(t=3.59 ; p<.001)$; toma de perspectiva $(U=4450 ; p$ $<.001)$; fantasía $(U=5254 ; p<.019)$ y malestar personal $(U=$ $5175 ; p<.013)$ que el grupo NO TD.

Tabla 4

Comparación de las medias de las pruebas de la teoría de la mente y las dimensiones de Empatía para ambos grupos

\begin{tabular}{lccc}
\hline & $t$ de Student & $U$ de Mann Whitney & $p$ \\
\hline Faux pas & 3.59 & & $<.001$ \\
Test de la mirada & 7.29 & & $<.001$ \\
& & & \\
IRI & & & .182 \\
- Preocupación empática & 1.34 & & $<.001$ \\
- Toma de perspectiva & & 4450.00 & .019 \\
- Fantasía & & 5254.00 & .013 \\
- Malestar personal & & 5175.00 & \\
\hline
\end{tabular}

Nota: IRI = Índice de Reactividad Interpersonal.

Tabla 3

Medias de las pruebas de teoría de la mente y dimensiones de la Empatía para el grupo TD y NO TD

\begin{tabular}{|c|c|c|c|c|c|c|}
\hline & \multicolumn{3}{|c|}{$\begin{array}{c}\text { TD } \\
(n=157)\end{array}$} & \multicolumn{3}{|c|}{$\begin{array}{l}\text { NO TD } \\
(n=82)\end{array}$} \\
\hline & $M$ & $D T$ & IC $95 \%$ & $M$ & $D T$ & IC $95 \%$ \\
\hline Faux pas & 62.40 & 16.61 & $59.78-65.58$ & 70.77 & 17.98 & $66.82-74.22$ \\
\hline Test de la Mirada & 16.39 & 9.66 & $15.66-17.13$ & 21.00 & 4.57 & $19.99-22.01$ \\
\hline \multicolumn{7}{|l|}{ IRI } \\
\hline - Toma perspectiva & 21.47 & 4.79 & $20.72-23.22$ & 23.91 & 3.97 & $23.04-24.79$ \\
\hline - Fantasía & 19.80 & 4.42 & $19.10-20.49$ & 21.10 & 3.67 & $20.29-21.90$ \\
\hline - Malestar personal & 20.32 & 4.67 & $19.59-21.06$ & 18.91 & 3.66 & $18.11-19.72$ \\
\hline - Preocupación empática & 22.86 & 4.17 & $22.20-23.52$ & 23.60 & 3.77 & $22.77-24.43$ \\
\hline
\end{tabular}

Nota $:$ IRI = Índice de Reactividad Interpersonal.

Con el fin de identificar la relación de la teoría de la mente, el funcionamiento intelectual y las dimensiones de la empatía en ambos grupos (TD y NO TD), se llevó a cabo un análisis de regresión logística binomial. El método usado fue el de adelante condicional, mediante el cual se introdujeron las variables independientes (CI Total, CI Verbal, CI Matrices, Faux pas, test de la mirada, fantasía, preocupación empática, malestar personal y toma de perspectiva) de acuerdo a la variable dependiente que fue la clasificación de los grupos (TD y NO TD). Se incluyó la variable CI como variable independiente, ya que en algunos estudios se ha comprobado que en los trastornos de conducta el bajo CI es una variable que ha mostrado una correlación significativa y como un factor predictivo en el desarrollo de conductas agresivas y antisociales en la infancia y la adolescencia (Baker, 2013; Faichild et al., 2009; Hill, 2002; Jones, Foster y Skuse, 2007; Mizock y Harkins, 2011).

Como se aprecia en la Tabla 5, el modelo de regresión logística incluyó finalmente tres variables. La presencia de estas variables es significativa de acuerdo con el estadístico chi cuadrado de Walt. La capacidad clasificatoria del modelo fue del $72.8 \%$.

Tabla 5

Análisis de regresión logística para la variable independiente (TD - NO TD) según las pruebas de coeficiente intelectual, teoría de la mente y empatía

\begin{tabular}{lcccccc}
\hline & $B$ & $E T$ & Wald & OR & \multicolumn{2}{c}{ IC 95\% } \\
& & & & Inferior & Superior \\
\hline CI Verbal & -.022 & .010 & 4.888 & .978 & .959 & .997 \\
Test de la mirada & -.204 & .038 & 29.029 & .816 & .758 & .879 \\
Toma de Perspectiva & -.091 & .035 & 6.751 & .913 & .852 & .978 \\
Constante & 8.671 & 1.411 & 37.785 & 5832.220 & & \\
\hline
\end{tabular}

Nota: $B=$ Coeficiente de regresión; $E T=$ Error típico; $O R=$ Odds Ratio; $I C=$ intervalo de confianza 
La bondad del modelo obtenido se sometió a contraste mediante la prueba de Hosmer y Lemeshow, arrojando un estadístico de chi cuadrado de 10.24 , con una probabilidad asociada $p=.248$, lo que significa una bondad de ajuste aceptable para el modelo. El signo de los coeficientes de regresión (B) indican el sentido de la relación entre cada una de las variables predictoras y la variable dependiente (TD y NO TD). Los valores del coeficiente de regresión para las tres variables incluidas en el modelo fueron negativas, dando lugar a odd ratio menores que 1; de esta forma el modelo de regresión mostró que el CI Verbal, el Test de la mirada y la dimensión de la empatía denominada Toma de perspectiva serían tomados como factores protectores para el TD.

\section{Discusión}

El presente estudio tuvo dos objetivos principales. El primero fue comparar la realización en funcionamiento intelectual, teoría de la mente y empatía en adolescentes con características de TD y adolescentes sin características de TD. El segundo objetivo fue establecer si dichas características podrían considerarse como factores predictivos de riesgo o de protección para el desarrollo de conductas disociales. Nuestras hipótesis sugieren que el grupo con características TD tendrá peores resultados en las tareas que evalúan cognición social mostrando déficits en las habilidades de la teoría de la mente y déficits en las capacidades empáticas en comparación con el grupo control. Por otro lado, formulábamos la hipótesis de que las ejecuciones en las diferentes pruebas de la teoría de la mente, CI y la empatía se configurarían como factores predictivos para el desarrollo de conductas disociales durante la adolescencia.

El principal hallazgo de este estudio, es que aparecieron diferencias muy significativas entre ambos grupos en las habilidades para "leer" estados emocionales en la mirada del otro y la sensibilidad social para detectar "meteduras de pata" en las interacciones sociales. El grupo TD mostró déficits significativos comparados con el grupo NO TD en estas habilidades reflejadas en los resultados en el test de la mirada y el Faux Pas. Estos hallazgos se corroboran con los resultados encontrados por varios autores (Fairchild et al., 2009; Gómez et al., 2010; Jones et al., 2007; Sharp, 2008), que además plantean que los déficits en estas habilidades de la teoría de la mente, afectan la capacidad del individuo para comprender la realidad del otro, acceder al reconocimiento de emociones, entender las normas de interacción social, generar procesos adecuados de regulación y autoregulación emocional y cognitiva necesarias para la interpretación de normas, reglas, principios y valores de una sociedad. De acuerdo a lo anterior y apoyados en los hallazgos de algunos autores (Eisenberg, 2009; Ellis, 1982; Jones et al., 2010; Miller y Eisenberg, 1988; Sharp et al., 2007), es posible establecer que los déficits en habilidades asociadas a la teoría de la mente pueden considerarse como predictores de conductas disociales ya que, si un individuo presenta déficits en las habilidades relacionadas con la teoría de la mente, estará limitado para discriminar las pistas afectivas y emocionales de los demás y tendrá serias dificultades para asumir la perspectiva del otro y responder ante sus necesidades; es decir, mostrará también dificultades para lograr vincularse empáticamente.

Un dato a destacar de este estudio es que no se hallaron diferencias significativas en la dimensión de preocupación empática en ambos grupos. Dolan y Fullan (2004) concluyeron en su estudio que los déficits en la teoría de la mente en individuos con conductas antisociales está más relacionado con su incapacidad para ponerse en el lugar del otro que con su falta de preocupación por el otro. Es decir, que los intentos espontáneos de adoptar el punto de vista del otro ante situaciones cotidianas o imaginadas, no necesariamente implican que los individuos logren una conexión afectiva. De hecho, el no encontrar déficits en la preocupación empática en el grupo TD, puede sugerir que aunque manifiestan conductas disociales y agresivas, estos jóvenes se dan cuenta de las consecuencias negativas de sus actos sobre los otros. Una explicación tentativa de este hallazgo es que la dimensión preocupación empática implica respuestas afectivas relacionadas con las consecuencias negativas que las conductas propias tienen sobre los otros. Esto quiere decir que los jóvenes del grupo TD son conscientes de que sus actos pueden perjudicar a otros, pero como sus déficits están más relacionados con la capacidad de adoptar la perspectiva del otro en contextos cotidianos o imaginarios, su preocupación empática no es un proceso afectivo suficiente para inhibir dichas conductas.

De acuerdo con lo anterior, es posible afirmar que los déficits hallados en la empatía pueden asociarse con las conductas disociales y agresivas de los jóvenes con características de TD y que los adolescentes sin características de TD que presentan normalidad en sus procesos empáticos poseen una probabilidad mayor para inhibir conductas agresivas y disociales. Esta conclusión está apoyada por los resultados de las investigaciones de Ellis (1982) y Miller y Eisenberg (1988) y generan datos importantes que aportan evidencias para validar nuestras hipótesis.

Nuestros resultados en cuanto al funcionamiento intelectual mostraron diferencias estadísticamente significativas entre ambos grupos. El grupo TD obtuvo puntuaciones más bajas en CI Total y Verbal que el grupo NO TD. No obstante, y contrario a lo que plantean algunos autores (Jones et al., 2007; Murray y Farrington, 2010), no se evidenció bajos desempeños intelectuales en el grupo TD, específicamente, en la subprueba de Matrices del K-BIT, los desempeños para los dos grupos fueron prácticamente iguales. Una explicación tentativa para este hallazgo, es que estas pruebas evalúan principalmente habilidades no verbales y flexibilidad aplicadas a estrategias de solución de problemas vinculados con habilidades espaciales. Dichas habilidades no parecen aportar de manera significativa al desarrollo de la empatía y la cognición social en nuestra muestra. Pero las capacidades del lenguaje si juegan un rol clave para 
expresar emociones de una manera precisa y adecuada, y también en la comprensión de las emociones expresadas por otros. Los déficits verbales pueden restringir las capacidades del individuo para expresar sus propias experiencias emocionales y su habilidad para procesar emocionalmente todo lo que sucede a su alrededor.

Nuestro segundo objetivo relacionado con establecer si las características empáticas, teoría de la mente y CI podrían considerarse como factores predictivos para el desarrollo de conductas disociales, fue logrado teniendo en cuenta el análisis de regresión logística binomial, según el cual no se encontraron dentro de las variables incluidas en el modelo, factores de riesgo para el desarrollo de conductas disociales; por el contrario, se sugiere que tanto las habilidades en la teoría de la mente como la de "leer" la mirada en los otros y aspectos de la empatía como la habilidad para asumir la perspectiva de otros, entender sus necesidades y sus estados mentales son factores de la cognición social que aumentarían la probabilidad de que un individuo manifieste conductas sociales apropiadas tanto en sus relaciones interpersonales como sociales. Por otro lado, en el modelo de regresión se encontró que el CI verbal también es considerado como un factor protector para el desarrollo de conductas disociales.

En consecuencia, se valida nuestra segunda hipótesis sobre si estas características de la teoría de la mente y la empatía serían entendidos como factores predictores de protección para el desarrollo de conductas disociales. De hecho los estudios mencionados previamente plantearon que las habilidades en cognición social como la teoría de la mente tienen gran validez predictiva para determinar la existencia de conductas disociales en niños y adolescentes, además, su adecuado desarrollo tiene efectos en las capacidades para inhibir conductas agresivas y antisociales. (Ellis, 1982; Fairchild et al., 2009; Miller y Eisenberg, 1988; Peets et al., 2010; Scourfield et al., 2004).

En conclusión, de acuerdo con nuestros resultados existen diferencias en las características de la teoría de la mente evaluadas en esta investigación. El grupo TD mostró mayores déficits en las habilidades para interpretar estados mentales y déficits en los procesos empáticos que el grupo NO TD. Si bien, no se pudo determinar si estos déficits eran considerados factores de riesgo, los análisis de los datos indicaron que mejores desempeños en la lectura de estados mentales en otros y la habilidad emocional para asumir la perspectiva de otro, junto con mejores ejecuciones en funcionamiento verbal, son considerados como factores predictivos que inhiben el desarrollo de conductas disociales en la muestra de adolescentes que evaluamos.

Por último, las implicaciones teórico - prácticas de este estudio van en la línea de profundización teórica y explicación sobre la naturaleza del TD durante la adolescencia, teniendo en cuenta el desarrollo empático y la cognición social; variables que han sido poco estudiadas de manera simultánea en poblaciones latinoamericanas. Desde una implicación práctica, se desprende la importancia de vincular las mediciones de la empatía y la teoría de la mente como ayuda diagnóstica en los trastornos de conducta y no solamente usar medidas conductuales y checklist basados en criterios clínicos. Por otro lado, el estudio de los factores de riesgo o protección para el desarrollo de trastornos de conducta, deben generar, por lo menos, una dirección terapéutica para prevenir o intervenir en las variables identificadas como predictoras del trastorno.

Para futuros estudios se hace necesario realizar descripciones y relaciones de variables como la empatía, el CI y la cognición social en el género femenino desde investigaciones longitudinales y comparativas con el género masculino, ya que son pocas las contribuciones que se han realizado en nuestro contexto. Sería recomendable también, ampliar esta investigación en adultos con trastornos de personalidad antisocial, con el fin de comparar en ambos períodos de desarrollo cómo cambian, qué los diferencia y especialmente, qué se podría hacer desde una postura terapéutica.

\section{Referencias}

1. American Psychiatry Asociation (2000). Diagnostic and statistical manual of mental disorders ( $4^{\mathrm{a}}$ edición revisada). Washington: Autor.

2. Baker, K. (2013). Conduct disorder in children and adolescents. Pediatrics and Child Health, 23, 24-29. http://dx.doi. org/10.1016/j.paed.2012.09.007

3. Baron - Cohen, S., O'Rioldan, M, Stone, V., Jones, R. y Plaisted, K. (1999). Recognition of Faux pas by normally developing children and children with Asperger Syndrome of High-functioning autism. Journal of Autism and Developmental Disorders, 29, 407-418. http://dx.doi. org/10.1023/A:1023035012436

4. Baron-Cohen, S., Wheelwright, S., Hill, J., Raste, Y. y Plumb, I. (2001). The reading the mind in the eyes. Test revised version: A study with normal adults, and adults with Asperger syndrome or high-functioning autism. Journal of Child Psychology and Psychiatry and Allied Disciplines, 42, 241-251. Recuperado de: http://docs.autismresearchcentre.com/papers/2001_BCetal_adulteyes.pdf

5. Baron - Cohen, S., Wheelwright, S. y Jolliffe, T. (1997). Is there a language of the eyes? Evidence from normal adults, and adults with autism or Asperger's Syndrome. Visual Cognition, 4, 311-331. http://dx.doi.org/10.1080/713756761

6. Benton, A.L., Hamsher, K.D.S., Varney, N. y Spreen, O. (1983). Contributions to neuropsychological assessment: A clinical manual. New York: Oxford University Press.

7. Blair, R.J. (2003). Facial expressions, their communicatory functions and neurocognitive substrates. Philosophical Transactions of the Royal Society of London B, 358, 561.572. http://dx.doi.org/10.1098/rstb.2002.1220

8. Davis, M. H. (1980). A multidimensional approach to individual differences in empathy. JSAS Catalog of Selected Documents in Psychology, 10, 85. 
9. Davis, M.H. (1983). Effects of dispositional empathy on emotional actions and helping: a multidimensional approach. Journal of Personality and Social Psychology, 44, 1113-1126. http://dx.doi.org/10.1111/j.1467-6494.1983.tb

10. Davis, M.H. (1983). Measuring individual differences in empathy: Evidence for a multidimensional approach. Journal of Personality and Social Psychology, 44, 113-126. http://dx.doi.org/10.1037/0022-3514.44.1.113

11. Dolan, M. y Fullam. R. (2004). Theory of mind and mentalizing ability in antisocial personality disorders with and without psychopathy. Psychological Medicine, 34, 1093 1102. http://dx.doi.org/10.1017/soo33291704002028

12. Eisenberg, N.D. (2009). Empathic responding: sympathy and personal distress. En: J. Decety y W. Ickes (Eds), The social neuroscience of empathy (pp. 71-83). Cambridge, Massachusetts: MIT Press.

13. Ekman, P. y Friesen, W.V. (1975). Pictures of facial affect. Palo Alto, C.A: Consulting Psychologist Press.

14. Ellis, P.L. (1982). Empathy: a factor in antisocial behavior. Journal of Abnormal Child Psychology, 10, 123 - 134. http://dx.doi.org/10.1007/BF00915957

15. Fairchild, G., Van Goozen, S., Calder, A., Stollery, S. y Goodyer, I. (2009). Deficits expressions recognitions in male adolescents with early-onset or adolescets - onset conduct disorder. The Journal of Child Psychology abd Psychiatry, 50, 627-636. http://dx.doi.org/10.1111/j.14697610.2008.02020x

16. Gómez, B.M., Arango, T.E., Molina, D. y Barceló, E. (2010). Características de la teoría en el trastorno disocial de la conducta. Psicología desde el Caribe, 26, 103-118. Recuperado de: http://rcientificas.uninorte.edu.co/index. php/psicologia/article/viewFile/1138/803

17. Goodman, R. (1997). The strengths and difficulties questionnaire: A research note. Journal of Child Psychology and Psychiatry and Allied Disciplines, 38, 581-586. http:// dx.doi.org/10.1111/j.1469-7610.1997.tb01545.x

18. Gregory, C., Lough, S., Stone, V., Erzingclioglu, S., Martin, L., Baron-Cohen, S. y Hodges, J.R. (2002). Theory of mind in patients with frontal variant frontotemporal dementia and Alzheimer's disease: Theoretical and practical implications. Brain, 125, 752 - 764. http://dx.doi.org/10.1093/ brain/awf079

19. Hill, J. (2002). Biological, psychological and social processes in the conduct disorders. Journal of Child Psychology and Psychiatry. 43, 133-164. http://dx.doi. org/10.1111/1469-7610.00007

20. Hughes, J.N., Meehan, B.T. y Cavell, T.A. (2004). Development and valiadation of a gender-balanced measure of aggression-relevant social cognition. Journal of Clinical Child and adolescent Psychology, 33, 292-302. http:// dx.doi.org/10.1207/s15374424jccp3302-11

21. Jolliffe, D. y Farrington, D. P. (2004). Empathy and offending: A systematic review and meta-analysis. Aggres- sion and Violent Behavior, 9, 441-476. http://dx.doi. org/10.1016/j.avb.2003.03.001

22. Jones, A.P., Forster, A.S. y Skuse, D. (2007). What do you think you're looking at? Investigating social cognition in Young offenders. Criminal Behaviour and Mental Health, 17, 101-106. http://dx.doi.org/10.1002/cbm.641

23. Jones, A.P., Happé, F.G., Gilbert, F., Brunett, S. y Viding, E. (2010). Feeling, caring, knowing: different type of empathy deficits in boys with psychopatic tendences and autism spectrum disorder. Journal of Child Psychology and Psychiatry, 51, 1188-1197. http://dx.doi.org/10.1111/j.14697610.2010.02280.x

24. Kaufman, A.S. y Kaufman, N.L. (1997). K-BIT. Test breve de inteligencia de Kaufman. Madrid: TEA Ediciones.

25. Miller, P.A. y Eisenberg, N. (1988). The relation of empathy to aggressive and externalizing/antisocial behavior. Psychological Bulletin, 103, 324 - 344. http://dx.doi. org/10.1037/0033-2909.103.3.324

26. Mizock, L. y Harkins, D. (2011). Diagnostic bias and conduct disorder: improving culturally sensitive diagnosis. Child \& Youth Services, 32, 243-253. http://dx.doi.org/10.1 $\underline{080 / 0145935 x .2011 .605315}$

27. Murray, J. y Farrington, D. (2010). Risks factors for conduct disorder and delinquency: key findings from longitudinal studies. The Canadian Journal of Psychiatry, 55, 633642. Recuperado de: http://publications.cpa-apc.org/media. php?mid=1051

28. Peets, K., Hodges, E.V. y Salmivalli, C. (2010). Actualization of social cognitions into aggressive behaviour toward disliked targets. Social Development, 20, 233-250. http:// dx.doi.org/10.1111/j.1457-9507.2010.00581.x

29. Román, F., Rojas, G., Román, N., Iturry, M., Romina, B., Bartoloni, A. y Allegri, R. (2012). Baremos del test de la Mirada en español en adultos normales de Buenos Aires. Revista Neuropsicología Latinoamericana, 4, 1-5. Recuperado de: http://neuropsicolatina.org/index.php/Neuropsicologia_Latinoamericana/article/view/108/91

30. Scourfield, J., Martin, N., Eley, T. C. y McGuffin, P. (2004). The gentic relationship between social cognition and conduct problems. Behaviour Genetics, 34, 377-383. http:// dx.doi.org/10.1023/B:BEGE.0000023643.49413.df

31. Sharp, C. (2008). Theory of mind and conducts problems in children: Deficits in reading the "emotions of the eyes". Cognition and Emotion, 22, 1149-1158. http://dx.doi. org/10.1080/02699930701667586

32. Sharp, C., Croudace, T. J. y Goodyer, I. M. (2007). A latent class analysis of a child-focused mentalising task in a community simple of 7-11 years olds. Social Development, 16, 181-202. http://dx.doi.org/10.1111/j.14679507.2007.00378.x

33. Sheehan, D.V., Lecrubier, Y., Harnett-Sheehan, K., Janavs, J., Weiller, E., Bonara, I. y Dunbar, G.C. (1997). Reliability and validity of the Mini International Neuropsychia- 
tric Interview (MINI): According of the SCID-P. European Psychiatry, 12, 232-241. http://dx.doi.org/10.1016/S09249338(97)83297-X

34. Stone, V.E., Baron-Cohen, S. y Knight, R.T. (1998). The frontal lobe contributions to Theory of Mind. Journal of Cognitive Neuroscience, 10, 640-656. http://dx.doi. org/10.1162/089892998562942

35. Weiss, B., Dodge, K.A., Bates, J.E. y Pettit, G.S. (1992). Some consequences of early harsh discipline: child aggression and a maladaptative social information processing style. Child Development, 63, 1321-1335. http://dx.doi. org/10.1111/j.1467.8624.1992.tb01697.x

Fecha de recepción: 6 de junio de 2013 Fecha de recepción de la versión modificada: 20 de septiembre de 2013 Fecha de aceptación: 28 de octubre de 2013 